\title{
Separation of disaccharide epimers, anomers and connectivity isomers by high resolution differential ion mobility mass spectrometry
}

\author{
Jordan Mastellone ${ }^{\#}$ K. M. Mohibul Kabir" and William A. Donald*
}

School of Chemistry, University of New South Wales, Sydney, NSW 2052, Australia.

\#JM and KMMK contributed equally

*To whom correspondence should be addressed: w.donald@unsw.edu.au 


\begin{abstract}
Glycans are ubiquitous, structurally diverse molecules that have specific and general roles involving metabolism, structure, energy storage, and cell-to-cell signalling. Functional specificity depends strongly on the complexity of structures that polysaccharides can adopt based on their subunit composition, length, extent of branching, glycosidic bond connectivity and anomeric configuration. However, the rapid, sensitive, and comprehensive characterization of glycan isomers can be challenging owing to limitations associated with their separation. Here, ten composition, anomeric and connectivity disaccharide isomers were separated and detected using high-resolution differential ion mobility-mass spectrometry (DMS-MS, also known as FAIMS). Focus was primarily directed to compositional isomers corresponding to epimers that differ by the axial or equatorial position of a single hydroxyl group. DMS resolving power was enhanced 15 -fold primarily by increasing the fraction of helium in the ion carrier gas and lowering the flow rate. DMS-MS of each disaccharide resulted in surprisingly complex and unique multi-peak spectra with up to ten fully and partially resolved peaks for $\beta$ 1,4-mannobiose (Man-1,4ß-Man). Each DMS spectrum has at least one differentiating peak that is not in the other spectra, indicating that DMS can be used to fully or partially resolve composition, configuration and connectivity isomers. Thus, DMS-MS has the potential to be used for identifying polysaccharide isomers in a spectral 'fingerprinting' approach. The integration of high-resolution DMS into high-throughput MS-based glycomics and glycoproteomics workflows may be useful in the future for improving the characterization of glycans and glycosylated biomolecules.
\end{abstract}




\section{Table of Contents Graphic}

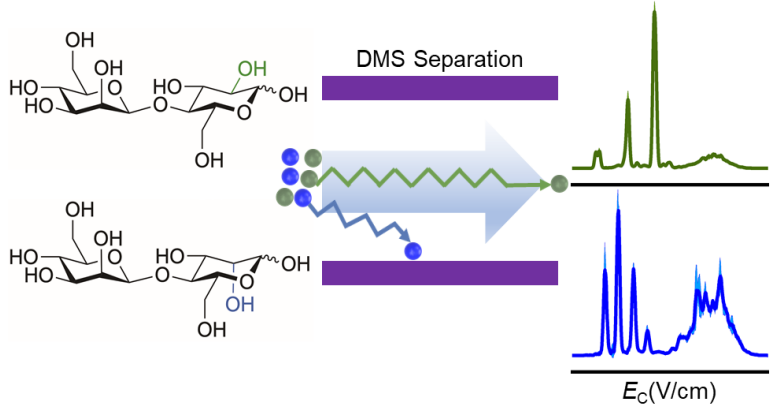


Glycans are of crucial importance in biological systems. ${ }^{1,2}$ Most cells in nearly all organisms are adorned with glycans that can have key roles in cell signaling and cell-to-cell recognition. Glycans contribute both generally and specifically to virtually all biological pathways ranging from explicit intracellular consequences to subtle phenotypic effects that only manifest at the whole-organism level. ${ }^{3}$ However, the chemical complexity of glycan structures is astounding owing to compositional isomerism of monosaccharide building blocks, branching, and the presence of stereocenters at glycosidic linkages. ${ }^{4}$ Glycans consist of monosaccharides linked by glycosidic bonds and exhibit many types of isomerism. Of the 75 known naturally occurring monosaccharide building blocks, 62 are found within ten general classes in which each class has the same elemental composition (composition isomers). ${ }^{5}$ For example, in the hexoses, there are eight naturally occurring monosaccharides with the same elemental composition $\left(\mathrm{C}_{6} \mathrm{H}_{12} \mathrm{O}_{6}\right)$. Each of these building blocks has an anomeric hemiacetal/acetal carbon which can adopt a discrete axial $(\alpha)$ or equatorial $(\beta)$ stereoisomeric configuration (configuration isomers), which can each connect to several possible carbons on an adjacent monosaccharide (connectivity isomers). In addition, there may be more than one glycosidic bond extending from a subunit, resulting in branched isomers. ${ }^{6}$ Even when limited to the nine common monosaccharides found in humans, over 15 million tetrasaccharides are possible. ${ }^{7}$ Thus, carbohydrates are considered more challenging to characterize than peptides and oligonucleotides.

The method of choice for certifying the purity of synthesized glycans is nuclear magnetic resonance (NMR) spectroscopy, which can provide highly detailed structural information. ${ }^{8}$ However, NMR typically requires milligram quantities of analyte, which can often exceed biologically relevant levels. The separation and detection of glycans at trace (femtomolar) levels is possible using fluorescent tagging in lectin- or antibody- binding assays, which has been proven useful for the targeted testing of hypotheses regarding glycan function. ${ }^{9}$ 
${ }^{10}$ Mass spectrometry based methods have the advantage that many different glycans can be detected rapidly and nearly simultaneously with high sensitivity in a single measurement. ${ }^{11}$

Although single-stage MS fundamentally cannot distinguish glycan isomers of equal $m / z$, tandem mass spectrometry $\left(\mathrm{MS}^{\mathrm{n}}\right)$ analysis can yield diagnostic fragment ions. Connectivity isomers can be distinguished by use of $\mathrm{MS}^{\mathrm{n}}$, either through several stages of collision-induced dissociation (CID) ${ }^{12}$ or ultra-violet photodissociation (UVPD). ${ }^{13}$ Infrared multiphoton dissociation (IRMPD) can result in fragment spectra largely equivalent to multistage or high energy $\mathrm{CID},{ }^{14-17}$ while electron-capture dissociation (ECD) can result in additional structurally informative dissociation pathways such as cross-ring cleavages. ${ }^{18,19}$ In matrix-assisted laser desorption ionization (MALDI), metastable precursor ions can undergo post-source fragmentation followed by CID fragmentation (in a TOF/TOF system) to obtain sequence information including connectivity isomerism. ${ }^{20-23}$ Nevertheless, differentiating constitutional and compositional isomer analysis by $\mathrm{MS}^{\mathrm{n}}$ is difficult because the resulting fragment ions have the exact same mass. Although relative fragment ion abundances may provide 'clues' for identifying constitutional and compositional stereoisomers, the rapid and unambiguous identification is highly challenging by $\mathrm{MS}^{\mathrm{n}}$ methods alone.

A range of methodologies have been used to separate glycans using liquid chromatography (LC) methods, including normal phase (polar), reverse phase (non-polar), anionic exchange, and antibody columns ${ }^{24}$ involving fine particle, high performance (HPLC) separations, sub-2.0 $\mu \mathrm{m}$ ultra-high performance (UHPLC) analysis, nano-flow column technology, and highly specific "chip" columns. ${ }^{25-27}$ Capillary electrophoresis integrated with ESI-MS can be used to separate $\alpha 2,3-$ and $\alpha 2,6-$ sialic acid connectivity isomers. ${ }^{28}$ However, like many other methods, the clear separation of configuration and composition isomers is challenging. Although the effects of such isomers in ubiquitous and well-studied glycans can 
often be minor, the influence of subtle isomeric differences in more unusual glycans can often be crucial and specific. 6,29

The separation of glycan isomers using ion mobility spectrometry (IMS)-based methods has attracted significant recent attention, particularly in the use of low-electric fieldbased methods (e.g. drift tube and travelling wave IMS) in which ions are rapidly separated primarily based on their mass, charge, and collision cross sections. ${ }^{30-34}$ The partial separation of disaccharides was achieved using travelling wave IMS (TWIMS), through derivatization using 1-phenyl-3-methyl-5-pyrazolone (PMP). ${ }^{35}$ Most recently, TWIMS has been combined with cryogenic infrared spectroscopy used to separate disaccharide isomers over long pathlengths. ${ }^{36}$ With such methods, the mobility of different ions depends linearly on the electric field strength and thus, ions are primarily separated based on any differences in their velocities through a carrier gas. ${ }^{37}$ However, many types of glycan isomer ions can have similar drift velocity owing to nearly identical collision cross sections and thus, cannot be readily resolved without the use of long ion pathlengths.

Differential ion mobility spectrometry (DMS), also known as field asymmetric ion mobility spectrometry (FAIMS), can separate ions based on their mobility differences under an alternating, asymmetric high $(>25 \mathrm{kV} / \mathrm{cm})$ and low electric field $(<10 \mathrm{kV} / \mathrm{cm}) .{ }^{38-50}$ Because the mobility of ions is non-linearly dependent on the electric field beyond $\sim 10 \mathrm{kV} / \mathrm{cm}$, ion separation can be more orthogonal to $m / z$ than lower-field IMS methods. The asymmetric field is generated by an alternating current, bisinusoidal potential that functions to radially disperse ions as they are carried between two electrodes by a gas. Only ions that have equal displacement in the high and low electric field segments are transmitted. A particular ion can be selectively transmitted by superimposing a compensation voltage $\left(V_{\mathrm{c}}\right)$ onto the asymmetric waveform corresponding to a compensation electric field $\left(E_{\mathrm{c}}\right)$, resulting in the effective filtering of ions. The use of planar DMS electrode geometries with relatively long channel 
lengths $(6.5 \mathrm{~cm})$ and high concentrations of low-molecular weight carrier gases has been demonstrated to have higher resolving power than alternative DMS methods. High resolution DMS can be used to separate peptide isomers that differ by the position of a single methyl group, ${ }^{51}$ protein protonation isomers, ${ }^{52}$ diastereomers, ${ }^{53,54}$ and isomeric glycopeptides.$^{55}$ Here, high resolution DMS is demonstrated to be capable of differentiating disaccharide isomers, including composition, configuration and connectivity isomers. Disaccharides can be considered fundamental models to reduce the complexity of glycan structure into a single isomeric subunit, in which two glycosydically-linked monosaccharides can differ based on epimeric, anomeric and connectivity isomerism. Such isomerism applies to all glycans and thus, disaccharides are useful for benchmarking the separation performance of different analytical platforms. An emphasis is placed on composition isomers because they have more closely related collision cross sections than configuration and connectivity isomers (owing to a conserved glycosidic bond structure) and have been more challenging to resolve by IMS and DMS.

\section{Experimental and methods}

Materials

4-O- $\alpha$-D-glucopyranosyl-D-glucose (1, Glc-1,4a-Glc), 4-O-( $\alpha$-D-galactopyranosyl)-

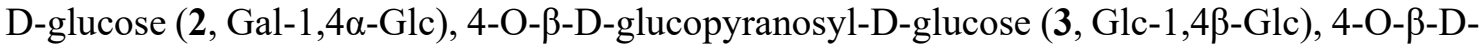

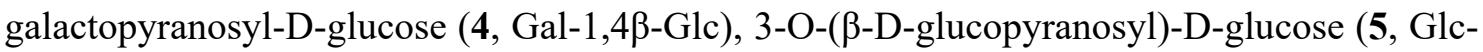

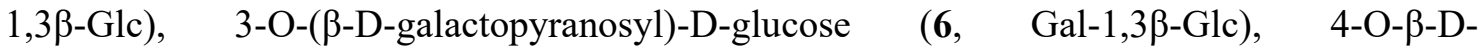
galactopyranosyl-D-mannose (7, Gal-1,4ß-Man), 4-O-( $\beta$-D-glucopyranosyl)-D-mannose (8,

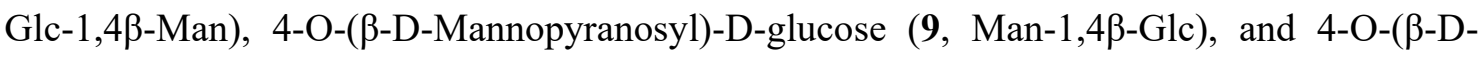

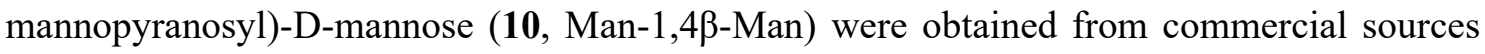
and used without further purification (see Table S1 for full details). Deionized water was obtained using a MilliQ water purification system (Merck, Darmstadt, Germany). Sodium 
chloride and HPLC-grade methanol were obtained from VWR (Radnor, PA, USA). High purity nitrogen and ultra-high purity helium gas cylinders (ALPHAGAZ ${ }^{\mathrm{TM}}$ ) were obtained from Air Liquide Australia Ltd. (Melbourne, VIC, Australia). Stock solutions were prepared by sonicating $1 \mathrm{mg}$ of each disaccharide or sodium chloride in $1 \mathrm{~mL}$ of deionized water, and dilution in HPLC-grade methanol $(90 \% \mathrm{v} / \mathrm{v})$ and deionized water $(10 \% \mathrm{v} / \mathrm{v})$ to a final disaccharide concentration of $1 \mathrm{mM}$ with $1 \mathrm{mM}$ sodium chloride. Relatively high concentrations were used to maximize ion signal and ensure that DMS spectra of sodiated disaccharide dimers can be readily obtained.

Differential ion mobility-mass spectrometry

All experiments were performed using a linear trap quadrupole MS (LTQ XL, Thermo Scientific, San Jose, CA, USA) integrated with a planar DMS (Heartland Mobility, Wichita, KS, USA). An electrodynamic ion funnel (Heartland Mobility, Wichita, KS, USA) was used as the interface between the DMS and the MS to increase ion transmission efficiencies. Liquid samples were infused by positive-mode ESI $(+3.4 \mathrm{kV}, 0.4 \mathrm{uL} / \mathrm{min})$ through a borosilicate capillary emitter (76 $\mu \mathrm{m}$ inner diameter) via a syringe pump (NE-300 by New Era Pump Systems, Farmingdale, NY, USA). Mass spectra were obtained from 100 to $1000 \mathrm{~m} / \mathrm{z}$ with 10 microscans and a maximum injection time of $20 \mu \mathrm{s}$. Ion transmission through the ion funnel was optimized to maximize ion signal (Table S2). In some specific cases, CID was performed using a $2 \mathrm{~m} / \mathrm{z}$ isolation window centered on the ion of interest using helium collision gas at a normalized collision energy of 5\% with $30 \mathrm{~ms}$ activation time.

The DMS instrument has two parallel, planar electrodes that are $65 \mathrm{~mm}$ long, $35 \mathrm{~mm}$ wide and separated by a $1.88 \mathrm{~mm}$ analytical gap. Ions generated by ESI are injected orthogonally into the gap through a cylindrical inlet $(2 \mathrm{~mm}$ diameter $)$ of a curtain plate $(+1.0$ $\mathrm{kV}$ ) and a second cylindrical inlet (1 mm diameter) in one of the DMS electrodes. The carrier gas composition ranged from 100 to $50 \%$ nitrogen gas mixed with 0 to $50 \%$ helium, and the 
flow rate was controlled from 0 to $2 \mathrm{~L} / \mathrm{min}$ using digital flow meters (MKS instruments, Andover, MA, USA). An in-house heater was used to apply up to $200{ }^{\circ} \mathrm{C}$ to the carrier gas tubing, consisting of $\sim 2 \mathrm{~m}$ of brass tubing coiled to a diameter of $40 \mathrm{~mm}$ and wrapped by silicone rubber heating tape, which was powered by a digital controller via temperature feedback from a type-K thermocouple. Pre-scan heating was performed for 3 hours prior to measurements to thermally equilibrate the DMS electrodes by applying up to $150{ }^{\circ} \mathrm{C}$ to the carrier gas tubing with the RF waveform dispersion voltage set to $4.00 \pm 0.01 \mathrm{kV}$. Carrier gas temperature was indirectly sampled using a type-K thermocouple and a resistance temperature detector (RTD) probe by measuring the temperature at the carrier gas tubing outlet connected to a partially sealed polypropylene tube of $25 \mathrm{~mm}$ diameter and $100 \mathrm{~mm}$ length (Figure $\mathrm{S} 1$ ).

DMS-MS spectra were obtained from the raw MS files by smoothing across 15 -scan intervals using Boxcar averaging in Xcalibur 2.2 QualBrowser (Thermo Fisher Scientific, San Jose, CA, USA). In the plotted spectra, shaded envelopes around median lines correspond to two standard deviations from the mean of 3-4 consecutive replicates. Figures were prepared using Origin Pro 2016b (OriginLab Corporation, Northampton, MA, USA).

\section{Results and Discussions}

\section{Selection of disaccharide isomers}

Ten disaccharide isomers (Figure 1) were chosen with an emphasis on composition isomers, which are epimers that differ by a single subunit. Such isomers generally have more closely related collision cross sections than connectivity and configuration isomers because their structures differ by only the axial or equitorial position of a single hydroxyl group (e.g. epimers $\mathbf{1}$ and 2). In Figure 1a, the six disaccharide isomers (1-6) correspond to three pairs of composition isomers that differ by a single monosaccharide subunit (epimer pairs 1/2, 3/4 and $\mathbf{5 / 6}$ ), two pairs of configuration isomers ( $\alpha$ or $\beta$ anomers, $\mathbf{1 / 3}$ and $\mathbf{2} / \mathbf{4}$ ) and two pairs of connectivity (glycosidic linkage) isomers (3/5 and 4/6). Four additional disaccharides (7-10) 
were chosen to provide six additional prototypical examples of compositional isomer pairs (epimers 4/7, 3/8, 9/10, 7/8, 3/9 and 8/10; Figure 1b). In Figure 1b, all adjacent isomers differ by only the axial or equatorial position of a single hydroxyl group (epimers). Overall, these ten disaccharides correspond to a total of nine pairs of epimers.
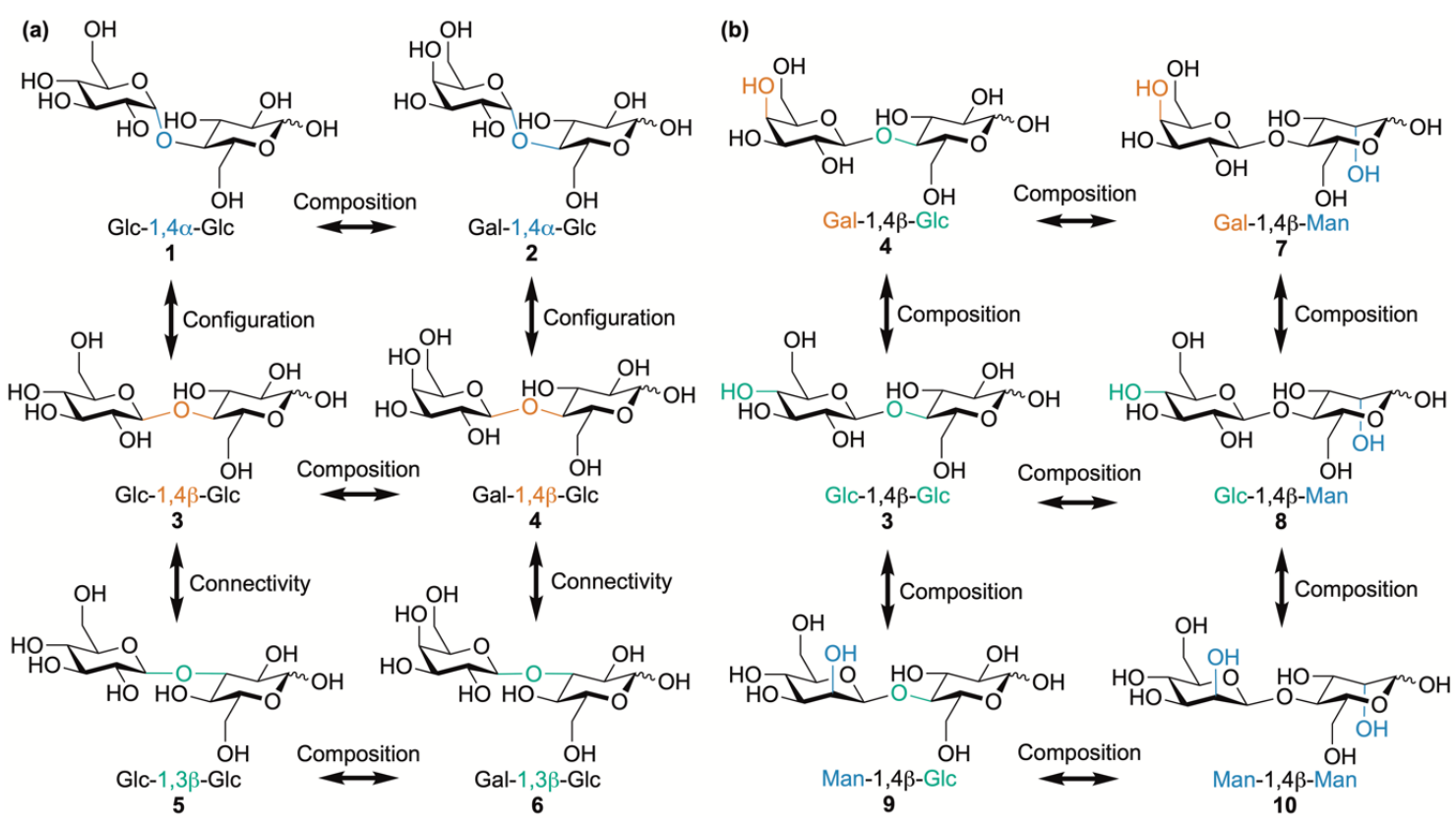

Figure 1. (a) Six of the ten disaccharide isomers investigated here (1-6). Composition (monosaccharide subunits), configuration ( $\alpha / \beta$ anomers) and connectivity (glycosidic linkage) isomers are indicated by arrows. (b) Six of the ten disaccharides that were investigated are composition isomers $(\mathbf{3}-\mathbf{4}, \mathbf{7}-\mathbf{8})$.

\section{Sodiated disaccharide dimer formation and dissociation}

ESI-MS and ESI-DMS-MS spectra of $1 \mathrm{mM} 4-\mathrm{O}-\beta-D$-glucopyranosyl-D-glucose in a 90:10\%(v/v) methanol:water solution containing $1 \mathrm{mM} \mathrm{NaCl}$ are shown in Figures S1a and $\mathrm{S} 1 \mathrm{~b}$, respectively. Relatively high concentrations were used to facilitate the formation of noncovalently bound ionic complexes, which can potentially be used to enhance ion separations in DMS. The ions were transmitted through the DMS device in 'transparency mode' with a carrier gas flow rate of $2 \mathrm{~L} / \mathrm{min}$ (i.e., RF and DC potentials were not applied to the DMS electrodes). In Figures $\mathrm{S} 1 \mathrm{a}$ and $\mathrm{S} 1 \mathrm{~b}$, the most abundant ion corresponds to the sodiated monomer $(\mathrm{m} / \mathrm{z} 365)$. A second ion formed in lower abundance $(10 \%)$ is assigned to the sodiated dimer $(\mathrm{m} / \mathrm{z} 707)$. For each disaccharide, CID of the ion at $\mathrm{m} / \mathrm{z} 707$ resulted in the formation of a major ion at $\mathrm{m} / \mathrm{z}$ 
365 , which is consistent with the precursor ion being assigned to the non-covalently bound sodiated dimer (e.g. Figure S1c). Thus, DMS-MS signals corresponding to $\mathrm{m} / z 365 \mathrm{can}$ arise both from: (i) the transmission of the sodiated monomer through the DMS device and (ii) higher ionic oligomers that are separated by DMS and then dissociate in the ion source to form the sodiated monomer, which is consistent with previously reported DMS-MS data for lithiated hexose ions. ${ }^{56}$

\section{Optimization}

The effects of the temperature, composition, and flow rate of the carrier gas on the DMS resolving power $\left(R_{\mathrm{DMS}}\right)$ was investigated next. $R_{\mathrm{DMS}}$ is defined as $\frac{V_{\mathrm{C}}}{w_{1 / 2}}$, where $v_{\mathrm{c}}$ is the compensation voltage (or compensation field) and $w_{1 / 2}$ is the full-width half maximum of ion peak. In principle, an increase in the difference between the high and low field mobility should result in an increase in resolving power, which can be obtained by using lower molecular weight carrier gases (e.g. helium) ${ }^{53,54}$ Here, the use of a DMS carrier gas with helium fractions higher than $\sim 50 \%$ in $\mathrm{N}_{2}(\mathrm{~g})$ resulted in the electrical breakdown of gas within the interelectrode gap $^{53,54}$ and thus, were not used. The use of lower carrier gas flow rates should also increase the resolving power with a corresponding increase in the ion residence time within the interelectrode gap, although at the expense of ion transmission efficiency. By controlling the temperature of the carrier gas, the signal stability was expected to improve, particularly for Type-C ions that are highly sensitive to ion-neutral clustering during the low-field portion of the waveform.

In Figure S3, DMS-MS spectra of sodiated disaccharide 4 as a function of the temperature applied to the carrier gas are shown. Across all temperatures, at least four peaks or partially resolved peaks are observed. As the temperature increases, the spectra change considerably. For example, at $40^{\circ} \mathrm{C}$, there are three peaks (labelled $a, b$, and $c$ ) observed between 32 and $64 \mathrm{~V} / \mathrm{cm}$, including an abundant peak at relatively low compensation field of 
32-43 V/cm (a) and a second major peak at higher fields (b; Figure S3). With increasing temperature, peak $a$ completely disappears by $100{ }^{\circ} \mathrm{C}$. In addition, a new peak $(d)$ emerges at $\sim 80-100^{\circ} \mathrm{C}$ and becomes quite abundant at higher temperatures. Thus, the DMS spectra are highly sensitive to the temperature of the carrier gas within the analytical gap, which is far lower than the applied temperature (Figure S1). Moreover, careful control of the temperature was necessary to obtain reproducible spectra under these conditions (data not shown). To further investigate whether the monomer signal can arise from dissociation of the dimer after DMS separation but prior to detection by MS, we compared the monomer and dimer spectra as a function of the temperature applied to the carrier gas (Figure S3). For a given temperature, the spectra for the monomer and dimer were remarkably similar in terms of the centroids and relative abundances with some key exceptions. In the dimer spectra, the corresponding peak $a$ was absent at all temperatures, unlike in the monomer spectra at lower temperatures. Thus, this lower field peak can be attributed to the transmitted monomer $[\mathrm{M}+\mathrm{Na}]^{+}$, whereas the higher field peak cluster is likely the monomer formed by in-source dissociation of different dimer conformations $[2 \mathrm{M}+\mathrm{Na}]^{+}$. Increasing the carrier gas temperature appears to suppress the monomer peak and alters the transmitted dimer conformations.

For all 10 compounds, as the fraction of helium in the carrier gas increased, the peaks generally narrowed (reducing $w_{1 / 2}$ ) and the peak centroids shifted to more positive values, resulting in an increase in $R_{\text {DMS }}$ for all 10 disaccharides (Figure 2). This observation is in line with the previous studies, which showed that the use of a low molecular weight gas in the carrier gas mixture can significantly improve the separation of ions because of the relatively higher mobility of ions in such gases. ${ }^{53,57-59}$ Overall, a 15-fold increase in $R$ DMs from $\sim 4$ to 57 was observed for sodiated disaccharide $3(365 \mathrm{~m} / \mathrm{z})$ when helium in the carrier gas was increased from 0 to $50 \%$. That is, four relatively abundant peaks were measured between 48 and $74 \mathrm{~V} / \mathrm{cm}$ (Figure S4b) with $50 \%$ helium whereas only a single peak was identified at $\sim 11$ 
V/cm using $0 \%$ helium in the carrier gas mixture (Figure S4a). Furthermore, this optimization resulted in the widths of the peaks narrowing by up to $50 \%$ and thus improving the overall RDMS.

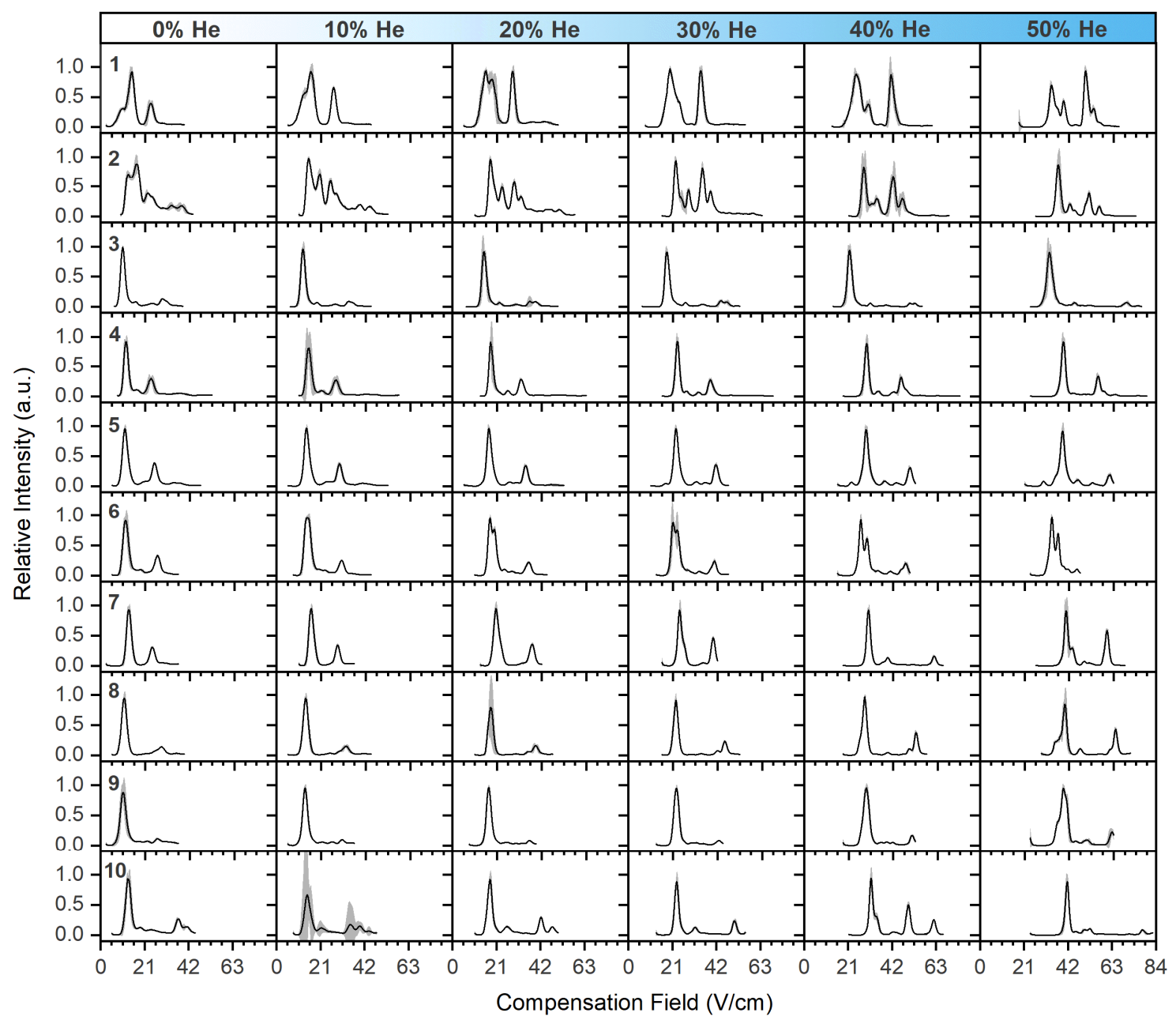

Figure 2. DMS-MS spectra of the sodiated disaccharides $[\mathrm{M}+\mathrm{Na}]^{+} \mathbf{1 - 1 0}(365 \mathrm{~m} / \mathrm{z})$ obtained using increasing amounts of helium in the carrier gas. The dispersion field was $22.3 \pm 0.1$ $\mathrm{kV} / \mathrm{cm}$. Data correspond to the mean of four replicates, and the shaded envelopes indicate the error range ( $95 \%$ confidence interval).

\section{DMS-MS spectra of disaccharide isomers}

Next, the ten disaccharide isomers were analyzed by DMS-MS. For clarity, the DMS spectra for all 9 compositional isomer pairs (epimers) and the two pairs of both configuration and connectivity isomers are shown in Figures 3 and 4, respectively, with expanded $x$-axis ranges. Overall, multiple DMS-MS peaks were observed between $40-83 \mathrm{~V} / \mathrm{cm}$ for all disaccharide isomers. In each case, composition, configuration, and connectivity isomers 
exhibited unique DMS-MS peak patterns in terms of the number of peaks, peak centroids and abundances (Figures 3 and 4). In Table S3, a summary of the peak patterns (peak centroids, widths, and abundances) obtained from Gaussian fitting of clearly resolved peaks is shown. The detection of up to 10 fully and partially resolved peaks for a single analyte (i.e. 10) is distinctive, and not generally observed in the case of low-field IMS or other DMS methods for small ionized molecules, ${ }^{32,33}$ thus enabling the potential identification of different isomers by using a 'fingerprint' approach.

The DMS-MS spectrum for each compositional isomer has one or multiple distinctive peaks and thus, can in principle be fully or partially resolved from its isomer pair (Figure 3 and Table S3). As an example, chosen at random, five peaks were measured for isomer $\mathbf{3}$ between 48.8 and $73.1 \mathrm{~V} / \mathrm{cm}$ whereas only two peaks were measured for isomer 4 at 53.7 and $56.6 \mathrm{~V} / \mathrm{cm}$, which corresponds to the baseline separation of multiple peaks between the two isomers. In some cases, partial resolution of epimers can be possible. For example, isomer 1 had three clearly resolved peaks at $49.8,51.3$ and $53.7 \mathrm{~V} / \mathrm{cm}$, unlike isomer 2 that also had three peaks but at different compensation fields $(48.9,50.4$ and $55.1 \mathrm{~V} / \mathrm{cm})$ and relative abundances. As a further example, isomer 9 has two well-resolved peaks at 49.3 and $60.2 \mathrm{~V} / \mathrm{cm}$ whereas the spectrum of its epimer $\mathbf{1 0}$ had three well-resolved, highly abundant peaks at 40.1, 45.4 and 51.6 $\mathrm{V} / \mathrm{cm}$. In contrast, isomer 8 has two major peaks at 47.6 and $64.1 \mathrm{~V} / \mathrm{cm}$ with a shoulder peak at $62.2 \mathrm{~V} / \mathrm{cm}$, which should thus enable the differentiation of $\mathbf{8}$ from its epimer $\mathbf{1 0}$ based on the DMS-MS spectra. Overall, the position of a single hydroxyl group (equatorial vs axial) can dramatically impact the DMS spectra of these ions under these conditions. 

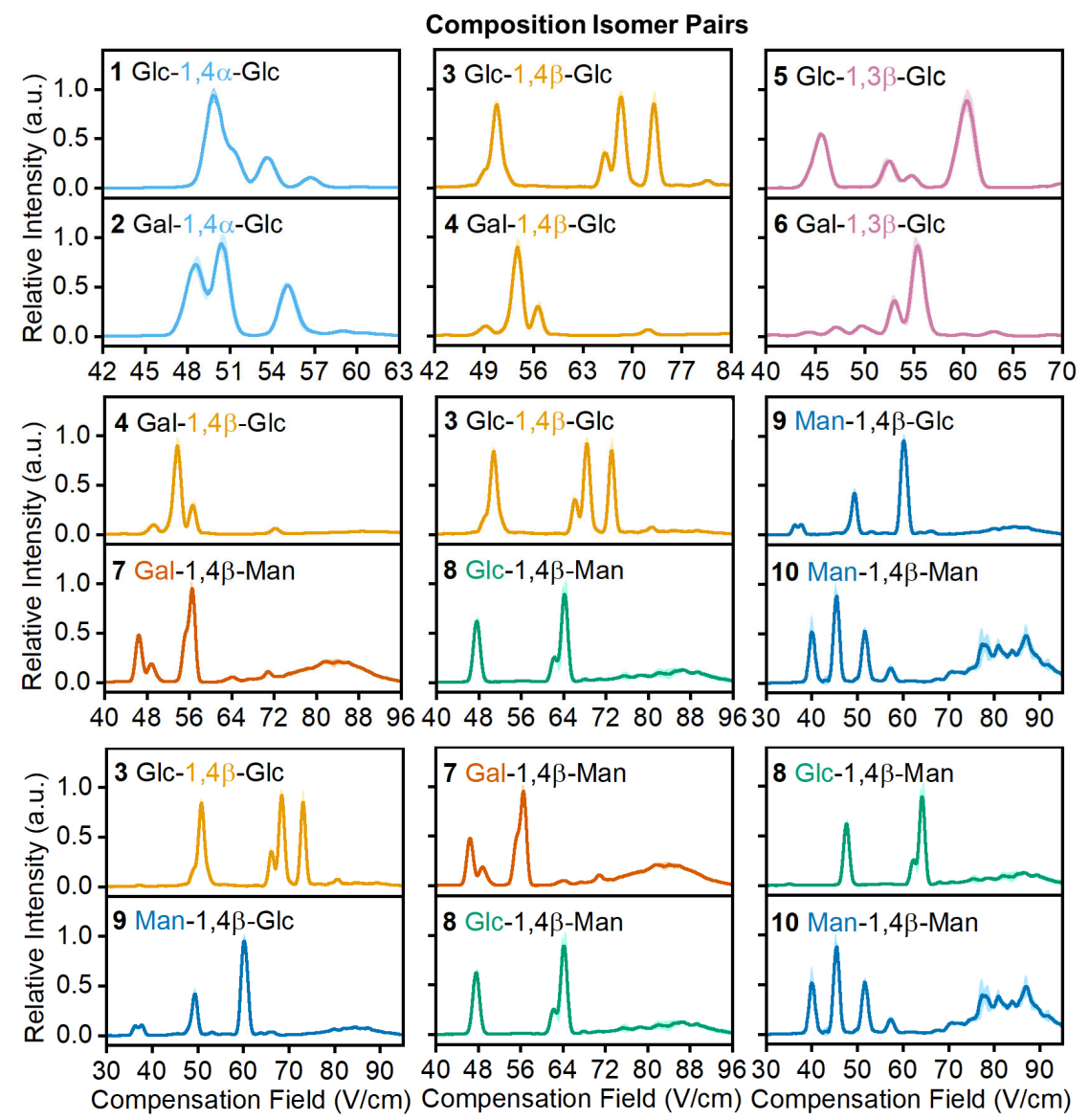

Figure 3. DMS-MS spectra of sodiated monomers $[\mathrm{M}+\mathrm{Na}]^{+}$for composition isomer pairs (epimers).

Like the compositional isomers, each of the configuration and connectivity pairs have multiple distinctive multi-peak patterns including at least one peak that can be resolved either fully or partially from its isomer pair (Figure 4). For the anomeric pair $\mathbf{1 / 3}$, isomer $\mathbf{3}$ has three DMS peaks between 66.1 and $73.1 \mathrm{~V} / \mathrm{cm}$ whereas isomer 1 had essentially no ion signal across this range of compensation fields, and three major peaks between 49.9 and $53.7 \mathrm{~V} / \mathrm{cm}$. For the connectivity pair $\mathbf{3 / 5}$, isomer $\mathbf{5}$ had four major peaks between 45.5 and $60.3 \mathrm{~V} / \mathrm{cm}$ compared to the spectrum for 3 which had three well resolved peaks from 66.1 to $73.1 \mathrm{~V} / \mathrm{cm}$. 

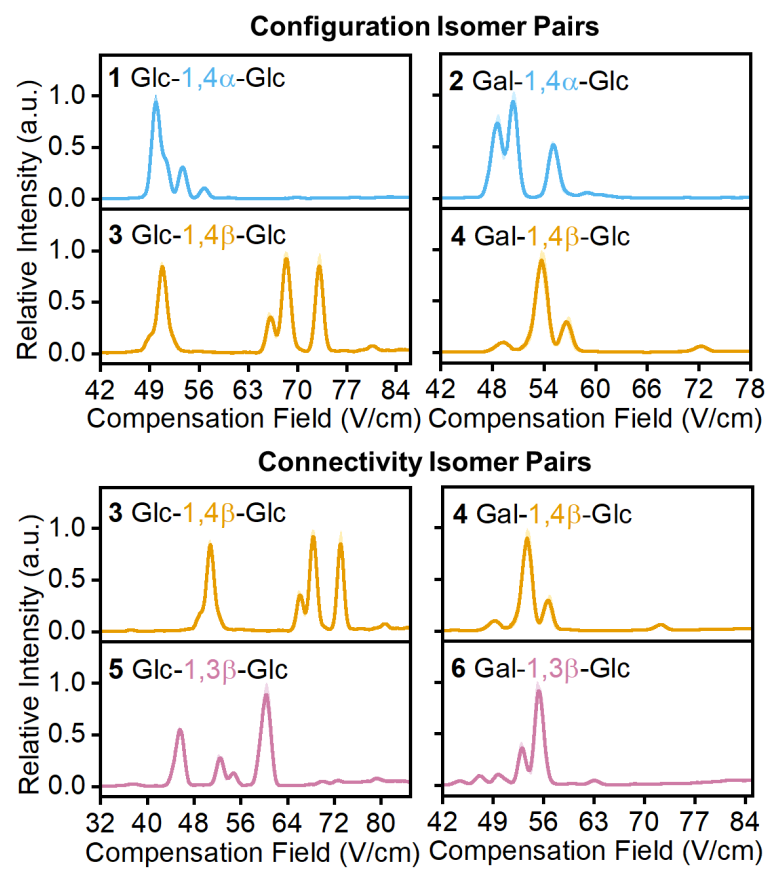

Figure 4. DMS-MS spectra of sodiated monomers $[\mathrm{M}+\mathrm{Na}]^{+}$for configuration (anomers) and connectivity isomer pairs.

By comparing the monomer and dimer DMS-MS spectra (Figure S5) for a given composition isomer, the number of peaks and peak centroids were broadly similar for both monomer and corresponding dimer spectra, but with some exceptions. For example, the number of peaks and corresponding compensation fields of the monomer and dimer spectra of configuration and connectivity isomers (Figure S5) were comparable for $\mathbf{2 ,} \mathbf{3}$ and $\mathbf{4}$. However, there were some major differences between the monomer and dimer spectra in some cases. For example, one additional peak for each of isomers $\mathbf{1}$ and $\mathbf{5}$ was observed in their dimer spectra compared to the corresponding monomer spectra. For isomers $\mathbf{2}$ and $\mathbf{6}$, the dimer spectra had two additional major peaks compared to the monomer spectra. Overall, the dimer spectra had the same or more peaks than the monomer spectra. Thus, both the monomer and dimer DMSMS spectra can potentially be used to differentiate isomers. The use of larger oligomeric cluster ions would likely result in even more complex isomer fingerprints.

The observation of several or more peaks in ion-mobility spectra has been previously reported for planar macroscale DMS-based analysis of moderately to relatively small ions such 
as glycopeptides ${ }^{55}$ and amino acid complexes. ${ }^{53,54}$ The origin of the multiple peaks in such spectra can be attributed to the presence of multiple conformers including charge solvation isomers during the separation process. For example, planar macroscale DMS-MS analysis of the proton-bound dimer of tryptophan and N-tert-butoxycarbonyl-O-benzyl-1-serine using $50 \%$ He and $\sim 22 \mathrm{kV} / \mathrm{cm}$ dispersion field resulted in three major peaks in the spectra, indicating the presence of different proton bound conformers including possible protonation isomers. ${ }^{53} \mathrm{DMS}$ MS analysis of T9 $\alpha$-GalNAc glycopeptide isomer using 50\% He and $\sim 27 \mathrm{kV} / \mathrm{cm}$ dispersion field resulted in the isomers having two distinctive peaks, corresponding to major and minor conformers. ${ }^{55}$ For type-C ions, such as those reported here, ion-neutral clustering in the low field portion of the waveform is particularly important and should be highly sensitive to conformation, including the local structure near the charge site. The relatively large number of hydroxyl groups on the carbohydrate ring function as charge acceptor sites for cation adduction, or the formation of transient neutral adducts. ${ }^{56}$ Additionally, the disaccharides are conformationally flexible and can readily adopt subtly different conformations, and the Oatoms can likely coordinate the sodium ion in many ways that are energetically competitive. Furthermore, 'glycans' as small as disaccharides have hydroxyl groups that may align clockwise or counterclockwise with each 6-membered monosaccharide ring, which are connected by a rotatable glycosidic bond. Thus, the ring structures may adopt one of many conformations, in addition to rotatable bonds around C6, and an anomeric carbon at the end of the glycan sequence. ${ }^{60}$

\section{Conclusion}

In this study, the separation and detection of a range of epimeric, connectivity and anomeric disaccharide isomers using high-resolution differential ion mobility-mass spectrometry (DMS-MS) is demonstrated. By use of an 'optimal' carrier gas composition and temperature, the resolving power of DMS was increased up-to 15-fold, and nine composition 
(monosaccharide subunits), two configuration ( $\alpha / \beta$ anomers) and two connectivity (glycosidic linkage) isomer pairs could be reproducibly differentiated. DMS-MS spectra of different disaccharides exhibited complex, multi-peak spectra with a single disaccharide resulting in upto ten peaks. Remarkably, the difference in the axial or equatorial position of a single hydroxyl group (i.e. in epimeric pairs) can dramatically affect the resulting DMS-MS spectral profiles. The complexity of peak distributions in the DMS-MS spectra also opens the possibility for identification based on database searching. A holistic "fingerprint" analysis could be used to determine unknown compounds through automated spectral matching and/or manual 'lookup' tables (e.g. Table S3). It is anticipated that the incorporation of DMS into MS-based glycomics and glycoproteomic instrumental platforms can potentially be used to enhance the characterization of more complex glycans and glycoconjugates.

\section{Acknowledgments}

The authors acknowledge Dr Ezaz Ahmed for collecting some of the ESI-MS data. KMMK and WAD acknowledge funding from Australian Research Council (DE190100986 and FT200100798, respectively).

\section{Author Information}

KMMK: orcid.org/0000-0003-3742-5742

WAD: orcid.org/0000-0002-6622-8193 


\section{References}

1. Karczewski, K. J.; Snyder, M. P., Integrative omics for health and disease. Nat. Rev. Genet. 2018, 19, 299-310.

2. Green, E. D.; Guyer, M. S., Charting a course for genomic medicine from base pairs to bedside. Nature 2011, 470, 204-213.

3. Cummings, R. D.; Pierce, J. M., The challenge and promise of glycomics. Chem. Biol. 2014, 21, 1-15.

4. McNaught, A. D.; Wilkinson, A., Compendium of chemical terminology. Blackwell Science Oxford: 1997; Vol. 1669.

5. Varki, A.; Cummings, R. D.; Aebi, M.; Packer, N. H.; Seeberger, P. H.; Esko, J.

D.; Stanley, P.; Hart, G.; Darvill, A.; Kinoshita, T., Symbol nomenclature for graphical representations of glycans. Glycobiology 2015, 25, 1323-1324.

6. Wopereis, S.; Lefeber, D. J.; Morava, E.; Wevers, R. A., Mechanisms in protein Oglycan biosynthesis and clinical and molecular aspects of protein O-glycan biosynthesis defects: a review. Clin. Chem. 2006, 52, 574-600.

7. Dove, A., The bittersweet promise of glycobiology. Nat. Biotechnol. 2001, 19, $913-$ 917.

8. De Reggi, M.; Capon, C.; Gharib, B.; Wieruszeski, J. M.; Michel, R.; Fournet, B., The glycan moiety of human pancreatic lithostathine: structure characterization and possible pathophysiological implications. Eur. J. Biochem. 1995, 230, 503-510.

9. $\quad$ Rudd, P. M.; Mattu, T. S.; Zitzmann, N.; Mehta, A.; Colominas, C.; Hart, E.;

Opdenakker, G.; Dwek, R. A., Glycoproteins: rapid sequencing technology for N-linked and GPI anchor glycans. Biotechnol. Genet. Eng. Rev. 1999, 16, 1-22.

10. Schedin-Weiss, S.; Winblad, B.; Tjernberg, L. O., The role of protein glycosylation in Alzheimer disease. FEBS J. 2014, 281, 46-62.

11. Merrill Jr, A.; Vu, M., Glycolipids. 2016.

12. Ashline, D. J.; Lapadula, A. J.; Liu, Y.-H.; Lin, M.; Grace, M.; Pramanik, B.;

Reinhold, V. N., Carbohydrate structural isomers analyzed by sequential mass spectrometry. Anal. Chem. 2007, 79, 3830-3842.

13. Devakumar, A.; Mechref, Y.; Kang, P.; Novotny, M. V.; Reilly, J. P., Identification of isomeric N-glycan structures by mass spectrometry with $157 \mathrm{~nm}$ laser-induced photofragmentation. J. Am. Soc. Mass Spectrom. 2008, 19, 1027-1040. 
14. Shi, S. D.-H.; Hendrickson, C. L.; Marshall, A. G.; Siegel, M. M.; Kong, F.; Carter, G. T., Structural validation of saccharomicins by high resolution and high mass accuracy fourier transform-ion cyclotron resonance-mass spectrometry and infrared multiphoton dissociation tandem mass spectrometry. J. Am. Soc. Mass Spectrom. 1999, 10, 1285-1290.

15. Xie, Y.; Lebrilla, C. B., Infrared multiphoton dissociation of alkali metal-coordinated oligosaccharides. Anal. Chem. 2003, 75, 1590-1598.

16. Zhang, J.; Schubothe, K.; Li, B.; Russell, S.; Lebrilla, C. B., Infrared multiphoton dissociation of O-linked mucin-type oligosaccharides. Anal. Chem. 2005, 77, 208-214.

17. Lancaster, K. S.; An, H. J.; Li, B.; Lebrilla, C. B., Interrogation of N-linked oligosaccharides using infrared multiphoton dissociation in FT-ICR mass spectrometry. Anal. Chem. 2006, 78, 4990-4997.

18. Budnik, B.; Haselmann, K.; Elkin, Y. N.; Gorbach, V.; Zubarev, R., Applications of electron- ion dissociation reactions for analysis of polycationic chitooligosaccharides in Fourier transform mass spectrometry. Anal. Chem. 2003, 75, 5994-6001.

19. Adamson, J. T.; Håkansson, K., Electron capture dissociation of oligosaccharides ionized with alkali, alkaline earth, and transition metals. Anal. Chem. 2007, 79, 2901-2910.

20. Stephens, E.; Maslen, S. L.; Green, L. G.; Williams, D. H., Fragmentation characteristics of neutral N-linked glycans using a MALDI-TOF/TOF tandem mass spectrometer. Anal. Chem. 2004, 76, 2343-2354.

21. Mechref, Y.; Kang, P.; Novotny, M. V., Differentiating structural isomers of sialylated glycans by matrix-assisted laser desorption/ionization time-of-flight/time-of-flight tandem mass spectrometry. Rapid Commun. Mass Spectrom. 2006, 20, 1381-1389.

22. Spina, E.; Sturiale, L.; Romeo, D.; Impallomeni, G.; Garozzo, D.; Waidelich, D.; Glueckmann, M., New fragmentation mechanisms in matrix-assisted laser desorption/ionization time-of-flight/time-of-flight tandem mass spectrometry of carbohydrates. Rapid Commun. Mass Spectrom. 2004, 18, 392-398.

23. Morelle, W.; Slomianny, M. C.; Diemer, H.; Schaeffer, C.; Dorsselaer, A. v.; Michalski, J. C., Fragmentation characteristics of permethylated oligosaccharides using a matrix-assisted laser desorption/ionization two-stage time-of-flight (TOF/TOF) tandem mass spectrometer. Rapid Commun. Mass Spectrom. 2004, 18, 2637-2649.

24. Zhao, J.; Simeone, D. M.; Heidt, D.; Anderson, M. A.; Lubman, D. M., Comparative serum glycoproteomics using lectin selected sialic acid glycoproteins with mass spectrometric analysis: application to pancreatic cancer serum. J. Proteome Res. 2006, 5, 1792-1802. 
25. Ruhaak, L. R.; Miyamoto, S.; Kelly, K.; Lebrilla, C. B., N-Glycan profiling of dried blood spots. Anal. Chem. 2012, 84, 396-402.

26. Chu, C. S.; Niñonuevo, M. R.; Clowers, B. H.; Perkins, P. D.; An, H. J.; Yin, H.; Killeen, K.; Miyamoto, S.; Grimm, R.; Lebrilla, C. B., Profile of native N-linked glycan structures from human serum using high performance liquid chromatography on a microfluidic chip and time-of-flight mass spectrometry. Proteomics 2009, 9, 1939-1951.

27. Hua, S.; An, H. J.; Ozcan, S.; Ro, G. S.; Soares, S.; DeVere-White, R.; Lebrilla, C. B., Comprehensive native glycan profiling with isomer separation and quantitation for the discovery of cancer biomarkers. Analyst 2011, 136, 3663-3671.

28. Kammeijer, G. S.; Jansen, B. C.; Kohler, I.; Heemskerk, A. A.; Mayboroda, O. A.; Hensbergen, P. J.; Schappler, J.; Wuhrer, M., Sialic acid linkage differentiation of glycopeptides using capillary electrophoresis-electrospray ionization-mass spectrometry. Sci. Rep. 2017, 7, 1-10.

29. Shao, L.; Haltiwanger, R., O-fucose modifications of epidermal growth factor-like repeats and thrombospondin type 1 repeats: unusual modifications in unusual places. Cell. Mol. Life Sci. 2003, 60, 241-250.

30. Morrison, K. A.; Clowers, B. H., Contemporary glycomic approaches using ion mobility-mass spectrometry. Curr. Opin. Chem. Biol. 2018, 42, 119-129.

31. Fenn, L. S.; McLean, J. A., Structural separations by ion mobility-MS for glycomics and glycoproteomics. In Mass Spectrometry of Glycoproteins, Springer: 2013; pp 171-194. 32. Hofmann, J.; Pagel, K., Glycan analysis by ion mobility-mass spectrometry. Angew. Chem. Int. Ed. 2017, 56, 8342-8349.

33. Hofmann, J.; Hahm, H.; Seeberger, P. H.; Pagel, K., Identification of carbohydrate anomers using ion mobility-mass spectrometry. Nature 2015, 526, 241-244.

34. Nagy, G.; Attah, I. K.; Garimella, S. V. B.; Tang, K.; Ibrahim, Y. M.; Baker, E. S.; Smith, R. D., Unraveling the isomeric heterogeneity of glycans: ion mobility separations in structures for lossless ion manipulations. Chem. Commun. 2018, 54, 11701-11704.

35. Yang, H.; Shi, L.; Zhuang, X.; Su, R.; Wan, D.; Song, F.; Li, J.; Liu, S., Identification of structurally closely related monosaccharide and disaccharide isomers by PMP labeling in conjunction with IM-MS/MS. Sci. Rep. 2016, 6, 1-9.

36. Ben Faleh, A.; Warnke, S.; Rizzo, T. R., Combining ultrahigh-resolution ionmobility spectrometry with cryogenic infrared spectroscopy for the analysis of glycan mixtures. Anal. Chem. 2019, 91, 4876-4882. 
37. Davis, E. J.; Grows, K. F.; Siems, W. F.; Hill Jr, H. H., Improved ion mobility resolving power with increased buffer gas pressure. Anal. Chem. 2012, 84, 4858-4865.

38. Gabryelski, W.; Froese, K. L., Rapid and sensitive differentiation of anomers, linkage, and position isomers of disaccharides using high-field asymmetric waveform ion mobility spectrometry (FAIMS). J. Am. Soc. Mass Spectrom. 2003, 14, 265-277.

39. Levin, D. S.; Vouros, P.; Miller, R. A.; Nazarov, E. G., Using a nanoelectrospraydifferential mobility spectrometer-mass spectrometer system for the analysis of oligosaccharides with solvent selected control over ESI aggregate ion formation. J. Am. Soc. Mass Spectrom. 2007, 18, 502-511.

40. Lane, C. S.; McManus, K.; Widdowson, P.; Flowers, S. A.; Powell, G.; Anderson, I.; Campbell, J. L., Separation of sialylated glycan isomers by differential mobility spectrometry. Anal. Chem. 2019, 91, 9916-9924.

41. Creese, A. J.; Cooper, H. J., Separation and identification of isomeric glycopeptides by high field asymmetric waveform ion mobility spectrometry. Anal. Chem. 2012, 84, 25972601.

42. Kabir, K. M. M.; Donald, W. A., Microscale differential ion mobility spectrometry for field deployable chemical analysis. Trends Analyt. Chem. 2017, 97, 399-427.

43. Winter, D. L.; Wilkins, M. R.; Donald, W. A., Differential Ion Mobility-Mass Spectrometry for Detailed Analysis of the Proteome. Trends Biotechnol. 2019, 37, 198-213.

44. Shvartsburg, A. A.; Creese, A. J.; Smith, R. D.; Cooper, H. J., Separation of peptide isomers with variant modified sites by high-resolution differential ion mobility spectrometry. Anal. Chem. 2010, 82, 8327-8334.

45. Shvartsburg, A. A.; Singer, D.; Smith, R. D.; Hoffmann, R., Ion mobility separation of isomeric phosphopeptides from a protein with variant modification of adjacent residues. Anal. Chem. 2011, 83, 5078-5085.

46. Shvartsburg, A. A.; Zheng, Y.; Smith, R. D.; Kelleher, N. L., Separation of variant methylated histone tails by differential ion mobility. Anal. Chem. 2012, 84, 6317-6320.

47. Shvartsburg, A. A.; Li, F.; Tang, K.; Smith, R. D., High-Resolution Field Asymmetric Waveform Ion Mobility Spectrometry Using New Planar Geometry Analyzers. Anal. Chem. 2006, 78, 3706-3714.

48. Shvartsburg, A. A.; Tang, K.; Smith, R. D., Modeling the resolution and sensitivity of FAIMS analyses. J. Am. Soc. Mass Spectrom. 2004, 15, 1487-1498. 
49. Campbell, J. L.; Kafle, A.; Bowman, Z.; Blanc, J. C. Y. L.; Liu, C.; Hopkins, W. S., Separating chiral isomers of amphetamine and methamphetamine using chemical derivatization and differential mobility spectrometry. Anal. Sci. Adv. 2020, 1, 233-244.

50. Anwar, A.; Psutka, J.; Walker, S. W. C.; Dieckmann, T.; Janizewski, J. S.; Larry Campbell, J.; Scott Hopkins, W., Separating and probing tautomers of protonated nucleobases using differential mobility spectrometry. Int. J. Mass spectrom. 2018, 429, 174181.

51. Winter, D. L.; Mastellone, J.; Kabir, K. M. M.; Wilkins, M. R.; Donald, W. A., Separation of Isobaric Mono- and Dimethylated RGG-Repeat Peptides by Differential Ion Mobility-Mass Spectrometry. Anal. Chem. 2019, 91, 11827-11833.

52. Zhang, J. D.; Donor, M. T.; Rolland, A. D.; Leeming, M. G.; Wang, H.; Trevitt, A. J.; Kabir, K. M. M.; Prell, J. S.; Donald, W. A., Protonation isomers of highly charged protein ions can be separated in FAIMS-MS. Int. J. Mass spectrom. 2020, 457, 116425. 53. Zhang, J. D.; Kabir, K. M. M.; Lee, H. E.; Donald, W. A., Chiral recognition of amino acid enantiomers using high-definition differential ion mobility mass spectrometry. Int. J. Mass spectrom. 2018, 428, 1-7.

54. Zhang, J. D.; Kabir, K. M. M.; Donald, W. A., Chapter Three - Ion-Mobility Mass Spectrometry for Chiral Analysis of Small Molecules. In Comprehensive Analytical Chemistry, Donald, W. A.; Prell, J. S., Eds. Elsevier: 2019; Vol. 83, pp 51-81.

55. Pathak, P.; Baird, M. A.; Shvartsburg, A. A., High-Resolution Ion Mobility Separations of Isomeric Glycoforms with Variations on the Peptide and Glycan Levels. $J$. Am. Soc. Mass Spectrom. 2020, 31, 1603-1609.

56. Campbell, M. T.; Chen, D.; Wallbillich, N. J.; Glish, G. L., Distinguishing Biologically Relevant Hexoses by Water Adduction to the Lithium-Cationized Molecule. Anal. Chem. 2017, 89, 10504-10510.

57. Zhang, J. D.; Kabir, K. M. M.; Donald, W. A., Metal-ion free chiral analysis of amino acids as small as proline using high-definition differential ion mobility mass spectrometry. Anal. Chim. Acta 2018, 1036, 172-178.

58. Shvartsburg, A. A.; Seim, T. A.; Danielson, W. F.; Norheim, R.; Moore, R. J.; Anderson, G. A.; Smith, R. D., High-definition differential ion mobility spectrometry with resolving power up to 500. J. Am. Soc. Mass Spectrom. 2013, 24, 109-114.

59. Shvartsburg, A. A.; Ibrahim, Y. M.; Smith, R. D., Differential ion mobility separations in up to $100 \%$ helium using microchips. J. Am. Soc. Mass Spectrom. 2014, 25, 480-489. 
60. Simons, J. P.; Jockusch, R. A.; ÇarÇabal, P.; Hünig, I.; Kroemer, R. T.; Macleod, N. A.; Snoek, L. C., Sugars in the gas phase. Spectroscopy, conformation, hydration, cooperativity and selectivity. Int. Rev. Phys. Chem. 2005, 24, 489-531. 